\title{
Asymptotic Performance Analysis of the $k$-th Best Link Selection over Wireless Fading Channels: An Extreme Value Theory Approach
}

\author{
Yazan H. Al-Badarneh, Costas N. Georghiades, Mohamed-Slim Alouini
}

\begin{abstract}
We consider a general selection-diversity (SD) scheme in which the $k$-th best link is selected from a number of links. We use extreme value theory (EVT) to derive simple closed-form asymptotic expressions for the average throughput, effective throughput and average bit error probability (BEP) for the $k$-th best link over various channel models that are widely used to characterize fading in wireless communication systems. As an application example, we consider the Weibull fading channel model and verify the accuracy of the derived asymptotic expressions through Monte Carlo simulations.
\end{abstract}

\section{INTRODUCTION}

Motivated by its simple implementation and low complexity, selection-diversity (SD) is an important diversity technique to enhance the performance of wireless communication systems. The theory of order statistics [1] is considered a powerful tool to analyze the performance of SD techniques. As a classical example, the theory of order statistics was used to analyze the performance of conventional SD schemes in which the link with the highest signal-to-noise ratio (SNR) is selected for transmission or reception from independent and identically distributed (i.i.d) links [2]. However, in practical communication systems the link with highest SNR may not be available for transmission or reception under given traffic conditions. Therefore, a more general SD scheme that features selection of the $k$-th best link ( $k$-th highest SNR or $k$-th largest order statistics) is of practical interest in wireless communication systems. Selection of the $k$-th best link has been considered in relay networks [3] [4] and cognitive radio networks [5].

Other applications of ordered statistics in wireless communication systems include analyzing the performance of generalized multiuser diversity schemes [6], minimum-selection generalized selection combining (MS-GSC) [7] and modeling the aggregate interference for centralized and decentralized selection schemes for the radio environment map (REM) approach in cognitive radio networks [8].

The effective throughput is defined as the maximum constant arrival rate that can be supported by a time-varying wireless channel under a statistical delay quality of service (QoS) constraint [9]. If no delay constraint is imposed, the effective throughput is the average (ergodic) throughput (capacity) of the corresponding wireless channel. In general, it is difficult to express in closed form the exact effective and average

Copyright(C)2015 IEEE. Personal use of this material is permitted. However, permission to use this material for any other purposes must be obtained from the IEEE by sending a request to pubs-permissions@iee.org. throughputs of SD schemes for various fading channel models. This is due to the complicated nature of the distribution of the SNR of the selected link. For example, the exact effective throughput of the conventional SD scheme in Rayleigh fading can be expressed as a logarithmic function of a finite sum of weighted Tricomi hypergeometric functions [10]. Furthermore, the average throughput of the conventional SD scheme in Rayleigh fading was expressed as a finite sum of weighted exponential integral functions [11].

The exact results for the effective throughput and average throughput derived in [10] and [11], respectively, are valid only for the conventional SD scheme in Rayleigh fading and it is hard to extend them for general SD schemes and different fading channels, such as Weibull, Gamma, $\alpha-\mu$ and GammaGamma. A recent attempt to analyze the average throughput of the $k$-th best link selection over a generalized Gamma ( $\alpha-$ $\mu$ ) fading channel was considered in [12], where the authors derived lower and upper bounds on the average throughput. However they did not investigate the effective throughput and average bit error probability (BEP).

In this paper, another approach based on extreme value theory (EVT) or extreme order statistics is used to analyze the effective throughput, average throughput and average BEP of the $k$-th best link over different fading channels. EVT was used to analyze the asymptotic average throughput (in the limit of large number of links) of the conventional SD scheme [13], [14]. EVT was also used to evaluate the average BEP of the conventional SD scheme [15], [16]. Our contribution in this paper is to utilize EVT to derive simple closed-form asymptotic and more intuitive expressions for the average throughput, effective throughput and average BEP of the $k$ th best link over various fading channel models, such as Weibull, Gamma, $\alpha-\mu$ and Gamma-Gamma. To the best of our knowledge, such analysis has not been considered in the literature before.

The rest of this paper is organized as follows. In Section II we discuss the system model. In Section III we discuss the average and effective throughputs of the $k$-th best link over Weibull, Gamma, $\alpha-\mu$ and Gamma-Gamma fading channels. In Section IV we analyze the average BEP. Section V includes numerical results and Section VI concludes.

\section{SYSTEM MODEL}

Consider a system in which the transmitter selects the $k$ th best link from $N$ i.i.d links to transmit information to the 
receiver. Let $h_{i}$ denote the complex channel gain of the $i$ th link between the transmitter and the receiver and $X_{i}$ the squared magnitude of the channel gain of the $i$-th link, where $i=1,2, \ldots, N$. The received signal can be expressed as

$$
y=h_{(N-k+1)} s+w,
$$

where $s$ is the complex valued symbol transmitted with (normalized) power $\rho, w$ is the circularly symmetric additive white Gaussian noise (AWGN) with zero mean and unit variance and $h_{(N-k+1)}$ is the flat fading channel gain of the $k$-th best link for $k=1,2, \ldots, N$. Then, the instantaneous SNR at the receiver side is $\rho X_{(N-k+1)}$ where $X_{(N-k+1)}=\left|h_{(N-k+1)}\right|^{2}$ and we assume the ordering $X_{(1)} \leq X_{(2)} \leq \ldots \leq X_{(N)}$. According to [1], the probability density function (PDF) of $X_{(N-k+1)}$ can be expressed in terms of the PDF, $f(x)$, and cumulative distribution function (CDF), $F(x)$, of $X_{i}$ as

$$
f_{X_{(N-k+1)}}(x)=k\left(\begin{array}{c}
N \\
k
\end{array}\right) f(x) F(x)^{N-k}(1-F(x))^{k-1} .
$$

Let $R_{i}=B \log _{2}\left(1+\rho X_{i}\right)$ denote the instantaneous throughput of the $i$-th link; then $R_{(N-k+1)}$ represents the instantaneous throughput of the selected link and can be evaluated as

$$
R_{(N-k+1)}=B \log _{2}\left(1+\rho X_{(N-k+1)}\right),
$$

where $R_{(1)} \leq R_{(2)} \leq \ldots \leq R_{(N)}$ and $B$ is the system bandwidth. Therefore, the average throughput of the selected link, $E\left[R_{(N-k+1)}\right]$, can be evaluated as

$$
E\left[R_{(N-k+1)}\right]=B \int_{0}^{\infty} \log _{2}(1+\rho x) f_{X_{(N-k+1)}}(x) d x .
$$

Assuming a block fading channel, the effective throughput that can be supported by a wireless system under a statistical QoS constraint described by the delay QoS exponent $\zeta$ is given by [9]

$$
\alpha(\zeta)=-\frac{1}{\zeta T} \log \left(E\left[e^{-\zeta T R}\right]\right), \zeta>0,
$$

where $R$ is a random variable which represents the instantaneous throughput during a single block and $T$ is the block length. $\zeta=0$ implies there is no delay constraint and the effective throughput is then the ergodic (average) throughput of the corresponding wireless channel.

Considering the $k$-th best link selection scheme, the effective throughput of the selected link, $\alpha(\theta, k, N)$, can be expressed as

$$
\alpha(\theta, k, N)=-\frac{1}{\theta} \log _{2}\left(E\left[e^{-\theta \ln (2) R_{(N-k+1)}}\right]\right)
$$

where $\theta=\zeta T$ and the expectation is taken over the distribution of $R_{(N-k+1)}$. Applying L'hospital's rule, one can show that $\lim _{\theta \rightarrow 0} \alpha(\theta, k, N)=E\left[R_{(N-k+1)}\right]$, as stated before.

In general, it is difficult to obtain closed form expressions for $E\left[R_{(N-k+1)}\right]$ and $\alpha(k, \theta, N)$ over various fading distributions. Therefore, in this paper, we consider another approach based on extreme value theory to analyze the average throughput and effective throughput over Weibull, Gamma, $\alpha-\mu$ and Gamma-Gamma fading channels.

\section{Throughrut ANALYSIS}

In this section, we combine the results from Sections 10.5 and 10.6 (distribution of the $k$-th extreme) of [1] in the following Proposition. We use this Proposition to derive the limiting distribution of the throughput of the $k$-th best link and to evaluate the average throughput and effective throughput.

Proposition 1 [1]: Let $Z_{(N)}$ denote the largest order statistic of $N$ i.i.d random variables with a common $\operatorname{CDF} F(z)$, where $Z_{(1)} \leq Z_{(2)} \leq \ldots \leq Z_{(N)}$. If $\frac{Z_{(N)}-a_{N}}{b_{N}}$, where $a_{N}$ and $b_{N}$ are normalizing constants, has a limiting $\mathrm{CDF}, G(z)$, then, for a fixed $k$ and $N \rightarrow \infty$, the limiting $\mathrm{CDF}$ of $\frac{Z_{(N-k+1)}-a_{N}}{b_{N}}$ is of the form

$$
G^{(k)}(z)=G(z) \sum_{j=0}^{k-1} \frac{[-\log (G(z))]^{j}}{j !} .
$$

Equivalently, the sequence $\frac{Z_{(N-k+1)}-a_{N}}{b_{N}}$ converges uniformly in distribution to a random variable $Z$, where the CDF of $Z$ is as in (7).

Let $X_{i}$ be i.i.d non-negative random variables for $i=$ $1,2, . . N$, where $X_{i}$ can be modeled as one of the following distributions: Exponential, Weibull, Gamma, $\alpha-\mu$ and Gamma-Gamma. In what follows we use Lemma 1 of [13] to obtain the limiting distribution of the largest order statistic, $X_{(N)}$. If the CDF of $X_{i}$ satisfies the conditions of this lemma, then the limiting distribution of the largest order statistic, $X_{(N)}$, is such that the sequence $\frac{X_{(N)}-\tilde{a}_{N}}{\tilde{b}_{N}}$ converges uniformly in distribution, for large $N$, to a normalized Gumbel random variable whose $\mathrm{CDF}, G(x)$, is given by

$$
G(x)=e^{-e^{-x}}, \quad-\infty<x<\infty .
$$

Furthermore, the normalizing constants $\tilde{a}_{N}$ and $\tilde{b}_{N}$ can be determined from the CDF of $X_{i}$ as [13]

$$
\begin{gathered}
\tilde{a}_{N}=F^{-1}\left(1-\frac{1}{N}\right), \\
\tilde{b}_{N}=F^{-1}\left(1-\frac{1}{N e}\right)-F^{-1}\left(1-\frac{1}{N}\right),
\end{gathered}
$$

where $F^{-1}(x)=\inf \{y: F(y) \geq x\}$. It was shown that the CDFs of Exponential, Gamma and Weibull distributions [13], $\alpha-\mu$ [17] and Gamma-Gamma [18] satisfy the conditions of Lemma 1 of [13]. Consequently, $\frac{X_{(N)}-\tilde{a}_{N}}{\tilde{b}_{N}}$ has a limiting distribution as expressed in (8). The normalizing constants $\tilde{a}_{N}$ and $\tilde{b}_{N}$ for the distributions of interest are tabulated in Table I.

Recall that $R_{i}=B \log _{2}\left(1+\rho X_{i}\right)$ is the instantaneous throughput of the $i$-th link, where $X_{i}$ can be modeled as one of the distributions referred to above. The limiting throughput distribution (LTD) Theorem of [13] provides a simpler approach to investigate the instantaneous throughput of the best link, $R_{(N)}$. The LTD Theorem indicates that the CDF of $R_{i}=B \log _{2}\left(1+\rho X_{i}\right)$ also satisfies the conditions of Lemma 1 of [13] and states that the sequence $\frac{R_{(N)}-a_{N}}{b_{N}}$ converges uniformly in distribution to a normalized Gumbel random 
TABLE I

NORMALIZING CONSTANTS FOR COMMON FADING DISTRIBUTIONS

\begin{tabular}{|c|c|c|c|}
\hline \multicolumn{2}{|c|}{\begin{tabular}{|l|} 
Distribution $\mid$ PDF \\
Exponential $e^{-x} u(x)$ \\
\end{tabular}} & \begin{tabular}{|l|}
$\tilde{a}_{N}$ \\
$\log (N)$
\end{tabular} & \begin{tabular}{|l|}
$b_{N}$ \\
1
\end{tabular} \\
\hline $\begin{array}{l}\text { Lxpoirntiac } \\
\text { Weibull }\end{array}$ & $\frac{\eta x^{\eta-1}}{x^{\eta}} e^{-x x^{\frac{1}{\alpha^{\prime}}}} u(x)$ & $\alpha(\ln (N))^{\frac{1}{\eta}}$ & $\frac{\alpha}{n}(\ln (N))^{-\frac{\eta-1}{\eta}}$ \\
\hline Gamma & $\frac{x^{m-1} e^{-x}}{\Gamma(m)} u(x)$ & $\begin{array}{l}\log (N) \underset{(m}{+}+(m) \\
O(\log (\log (\log (N))))\end{array}$ & Fin \\
\hline \begin{tabular}{|l} 
Gamma- \\
Gamma \\
{$[18]$}
\end{tabular} & $\begin{array}{l}\frac{2(k m) \frac{k+m}{2}}{\Gamma(m) \Gamma(k)}(x)^{\frac{k+m}{2}-1} \\
K_{k-m}[2 \sqrt{k m x}] u(x)\end{array}$ & $\frac{(\log (N))^{2}}{4 k m}+O(\log (N) \log (\log (N)))$ & 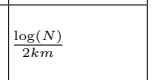 \\
\hline$\alpha-\mu[17]$ & $\frac{\alpha \mu^{\mu} x^{\frac{\alpha \mu}{2}-1} \exp \left(-\mu \frac{\frac{\alpha}{\frac{\alpha}{2}}}{\zeta \frac{\alpha}{2}}\right)}{2 \zeta^{\frac{\alpha \mu}{2}} \Gamma(\mu)} u(x)$ & $\left\{\begin{array}{l}\zeta\left(\frac{\log (K)}{\mu}\right)^{\frac{2}{\alpha}}+ \\
O\left(\left(\frac{\log (N)}{\mu}\right)^{\frac{2}{\alpha}-1} \log (\log (N))\right)\end{array}\right.$ & $\frac{2 \zeta\left(\frac{\log (N)}{\mu}\right)^{-1+\frac{2}{\alpha}}}{\alpha \mu}$ \\
\hline
\end{tabular}

variable with CDF, $G(x)$, as in (8). The normalizing constants $a_{N}$ and $b_{N}$ associated with $R_{(N)}$ can be obtained from $\tilde{a}_{N}$ and $\tilde{b}_{N}$ as [13]

$$
\begin{gathered}
a_{N}=B \log _{2}\left(1+\rho \tilde{a}_{N}\right), \\
b_{N}=B \log _{2}\left(1+\frac{\rho \tilde{b}_{N}}{1+\rho \tilde{a}_{N}}\right) .
\end{gathered}
$$

While we focus next on the case where $X_{i}$ can be modeled as Exponential, Weibull, Gamma, $\alpha-\mu$ and Gamma-Gamma to derive the average throughput, effective throughput and average BEP for the $k$-th best link, it should be noted that the following derived results are valid for any random variable $X_{i}$ whose CDF satisfies the conditions of Lemma 1 of [13].

\section{A. Average Throughput}

The LTD Theorem states that the sequence $\frac{R_{(N)}-a_{N}}{b_{N}}$ converges in distribution to a normalized Gumbel random variable. Using this and Proposition 1, we characterize the limiting distribution of the throughput of the $k$-th best link, $R_{(N-k+1)}$, in the following proposition.

Proposition 2: For a fixed $k$ and $N \rightarrow \infty$, the sequence $\frac{R_{(N-k+1)}-a_{N}}{b_{N}}$ converges uniformly in distribution to a random variable $X$ with $\mathrm{CDF}$ given by

$$
G^{(k)}(x)=e^{-e^{-x}} \sum_{j=0}^{k-1} \frac{e^{-j x}}{j !}, \quad-\infty<x<\infty .
$$

Furthermore, the average throughput can be approximated as

$$
E\left[R_{(N-k+1)}\right] \approx a_{N}-\psi(k) b_{N},
$$

where $\psi(x)$ is the digamma function.

Proof: The sequence $\frac{R_{(N)}-a_{N}}{b_{N}}$ converges uniformly in distribution to a normalized Gumbel random variable with CDF $G(x)=e^{-e^{-x}}, \quad-\infty<x<\infty$, where $a_{N}$ and $b_{N}$ are as defined in (11) and (12), respectively. Using Proposition 1 , it follows that for a fixed $k$ and $N \rightarrow \infty$, the sequence $\frac{R_{(N-k+1)}-a_{N}}{b_{N}}$ converges uniformly in distribution to a random variable $X$ with $\operatorname{CDF} G^{(k)}(x)$ as expressed in (13).

To prove (14) of Proposition 2, we first prove the following Lemma in the Appendix.
Lemma : If $\frac{R_{(N-k+1)}-a_{N}}{b_{N}}$ converges in distribution to a random variable $X$ whose $\mathrm{CDF}$ as expressed in (13) then for any positive real number $p$, we have

$$
E\left[\left(\frac{R_{(N-k+1)}-a_{N}}{b_{N}}\right)^{p}\right] \rightarrow E\left[X^{p}\right],
$$

for a fixed $k$ and $N \rightarrow \infty$. Furthermore, $E[X]=-\psi(k)$ and the moment generating function (MGF) associated with $X, \mathcal{M}_{X}(t)=E\left[e^{t X}\right]=\Gamma(k-t) /(k-1) !, k>t$.

It follows from this Lemma that for $p=1$, $E\left[\left(\frac{R_{(N-k+1)}-a_{N}}{b_{N}}\right)\right] \rightarrow E[X]=-\psi(k)$, for a fixed $k$ and $N \rightarrow \infty$. Therefore, the average throughput can be approximated as

$$
E\left[R_{(N-k+1)}\right] \approx a_{N}-b_{N} \psi(k) .
$$

As special case, if $k=1, \psi(1)=-\gamma$ (Euler's constant) and thus $E\left[R_{(N)}\right] \approx a_{N}+\gamma b_{N}$, which is exactly the same expression derived in [13]. Since $\psi(k)>0$, for $k>1$ and integer $k$, it follows that $E\left[R_{(N-k+1)}\right]<E\left[R_{(N)}\right]$, for $k>$ 1. It should be noted that the result in Proposition 2 can be used to evaluate the outage throughput of the $k$-th best link. Given a rate $R_{0}, P_{\text {out }}\left(R_{0}\right)$ can be approximated as

$$
\begin{aligned}
P_{\text {out }}\left(R_{0}\right) & \triangleq \operatorname{Pr}\left\{R_{(N-k+1)} \leq R_{0}\right\} \\
& =\operatorname{Pr}\left\{\frac{R_{(N-k+1)}-a_{N}}{b_{N}} \leq \frac{R_{0}-a_{N}}{b_{N}}\right\} \\
& \approx \operatorname{Pr}\left\{X \leq \frac{R_{0}-a_{N}}{b_{N}}\right\}=G^{(k)}\left(\frac{R_{0}-a_{N}}{b_{N}}\right),
\end{aligned}
$$

where $G^{(k)}(x)$ is as expressed in (13).

\section{B. Effective Throughput}

In this subsection, we use the result from Proposition 2 to analyze the effective throughput of the $k$-th best link as in the following proposition.

Proposition 3: The effective throughput of the $k$-th best link, $\alpha(\theta, k, N)$, can be approximated as

$$
\alpha(\theta, k, N) \approx a_{N}-\frac{1}{\theta} \log _{2}\left(\frac{\Gamma\left(\theta \ln (2) b_{N}+k\right)}{(k-1) !}\right)
$$

for fixed $k, \theta>0$ and $N \rightarrow \infty$, where $\Gamma(\cdot)$ is the gamma function.

Proof: Invoking (6), the term $E\left[e^{-\theta \ln (2) R_{(N-k+1)}}\right]$ represents the MGF associated with the random variable $R_{(N-k+1)}$, $\mathcal{M}_{R_{(N-k+1)}}(t)$, at $t=-\theta \ln (2)$. Since $\theta>0$, then we are always interested in the case $t \in(-\infty, 0)$. Applying the result from Theorem 2 of [19], which implies that if $\mathcal{M}_{R_{(N-k+1)}}(t)$ exists for all $t \in(-\infty, 0)$ and the sequence $Y_{N, k}=\frac{R_{(N-k+1)}-a_{N}}{b_{N}}$ converges uniformly in distribution to a random variable $X$ with CDF as expressed in (13) and MGF of $\mathcal{M}_{X}(t)$ which exists for all $t \in(-\infty, 0)$, then for a fixed $k, \lim _{N \rightarrow \infty} \mathcal{M}_{Y_{N, k}}(t)=\mathcal{M}_{X}(t)$ for all $t \in(-\infty, 0)$, where $\mathcal{M}_{Y_{N, k}}(t)$ is the MGF associated with the random variable $Y_{N, k}$. 
It is obvious that $\mathcal{M}_{X}(t)=\Gamma(k-t) /(k-1)$ ! exists for all $t \in(-\infty, 0)$. However, to show that $\mathcal{M}_{R_{(N-k+1)}}(t)$ exists for all $t \in(-\infty, 0)$, we use Lemma 1.7.2. of [20], with $\mathrm{g}(\mathrm{x})=$ $(1+x)^{t}, x \geq 0$ and $t \in(-\infty, 0)$; we have

$$
\begin{aligned}
\mathcal{M}_{R_{(N-k+1)}}(t) & =E\left[e^{t R_{(N-k+1)}}\right] \\
& =E\left[e^{\frac{t B}{\ln (2)} \log \left(1+X_{(N-k+1)}\right)}\right] \\
& =E\left[\left(1+X_{(N-k+1)}\right)^{\frac{t B}{\ln (2)}}\right] \\
& \leq \frac{N !}{(k-1) !(N-k) !} E\left[\left(1+X_{i}\right)^{\frac{t B}{\ln (2)}}\right] .
\end{aligned}
$$

Since $X_{i}$ is non-negative random variable and it can be modeled as Exponential, Weibull, Gamma, $\alpha-\mu$ and GammaGamma, it follows that $E\left[\left(1+X_{i}\right)^{\frac{t B}{\ln (2)}}\right]<\infty$, for all $t \in$ $(-\infty, 0)$. Using Theorem 2 of [19], we have

$$
\lim _{N \rightarrow \infty} E\left[e^{t\left(\frac{R_{(N-k+1)}-a_{N}}{b_{N}}\right)}\right]=E\left[e^{t X}\right]=\frac{\Gamma(k-t)}{(k-1) !},
$$

for all $t \in(-\infty, 0)$. Therefore,

$$
\begin{aligned}
& E\left[e^{\left.-\theta \ln (2) R_{(N-k+1)}\right]}\right. \\
& =E\left[e^{-\theta \ln (2)\left[b_{N}\left(\frac{R_{(N-k+1)}-a_{N}}{b_{N}}\right)+a_{N}\right]}\right] \\
& \approx e^{-\theta \ln (2) a_{N}} E\left[e^{-\theta \ln (2) b_{N} X}\right] \\
& =\frac{e^{-\theta \ln (2) a_{N}} \Gamma\left(k+\theta \ln (2) b_{N}\right)}{(k-1) !}
\end{aligned}
$$

for a fixed $k$ and $N \rightarrow \infty$. Substituting (21) in (6) we reach (18). It is interesting to observe that if $\theta \rightarrow 0$, the effective throughput in (18) becomes the average throughput in (16). In order to show this, we apply L'hospital's rule and use $\frac{\partial}{\partial x} \log (\Gamma(x))=\psi(x)$; then we have

$$
\begin{aligned}
\lim _{\theta \rightarrow 0} \alpha(\theta, k, N) & \approx a_{N}-b_{N} \lim _{\theta \rightarrow 0} \psi\left(\theta \ln (2) b_{N}+k\right) \\
& =a_{N}-b_{N} \psi(k)=E\left[R_{(N-k+1)}\right] .
\end{aligned}
$$

\section{Asymptotic Average Bit Error Probability}

We consider a general class of modulation schemes whose conditional BEP, $P_{e}$, is given by [2]

$$
P_{e}=C e^{-g Y},
$$

where $C$ and $g$ are positive constants and $Y$ is a random variable which represents the instantaneous received SNR. The average BEP, $\overline{P_{e}}$, can be expressed as

$$
\overline{P_{e}}=C E\left[e^{-g Y}\right] \text {. }
$$

Considering the $k$-th best link selection scheme, the instantaneous received SNR is $Y=\rho X_{(N-k+1)}$, as stated earlier. Therefore, the average BEP of the $k$-th best link is $\overline{P_{e}}=C E\left[e^{-g \rho X_{(N-k+1)}}\right]$. Using the fact that the sequence $\frac{X_{(N)}-\tilde{a}_{N}}{\tilde{b}_{N}}$ converges in distribution to a normalized Gumbel random variable along with Proposition 1, we derive the average BEP of the $k$-th best link in the following proposition.
Proposition 4: The average BEP of the $k$-th best link, $\overline{P_{e}}$, can be approximated as

$$
\overline{P_{e}} \approx C e^{-g \rho \tilde{a}_{N}} \frac{\Gamma\left(k+g \rho \tilde{b}_{N}\right)}{(k-1) !}
$$

for fixed $k$ and $N \rightarrow \infty$.

Proof: Using moment generating function approach, the average BEP of the $k$-th best link can be expressed as [2]

$$
\overline{P_{e}}=C E\left[e^{-g \rho X_{(N-k+1)}}\right]=C \mathcal{M}_{X_{(N-k+1)}}(-g \rho),
$$

where $\mathcal{M}_{X_{(N-k+1)}}$ represents the MGF associated with the random variable $X_{(N-k+1)}$. Using the fact that the sequence $\frac{X_{(N)}-\tilde{a}_{N}}{\tilde{b}_{N}}$ converges uniformly in distribution for large $N$ to a normalized Gumbel random variable, it follows that, from Proposition 1, the sequence $\frac{X_{(N-k+1)}-\tilde{a}_{N}}{\tilde{b}_{N}}$ converges uniformly in distribution to the random variable $X$ with $\mathrm{CDF}$ as expressed in (13). Following similar analysis in the proof of the effective throughput, we infer that the MGF of $\frac{X_{(N-k+1)}-\tilde{a}_{N}}{\tilde{b}_{N}}$ converges to the MGF of $X$ and therefore $\overline{P_{e}}$ can be approximated as

$$
\begin{aligned}
\overline{P_{e}} & =C E\left[e^{-g \rho X_{(N-k+1)}}\right] \\
& \approx C e^{-g \rho \tilde{a}_{N}} E\left[e^{-g \rho \tilde{b}_{N} X}\right]=C e^{-g \rho \tilde{a}_{N}} \frac{\Gamma\left(k+g \rho \tilde{b}_{N}\right)}{(k-1) !},
\end{aligned}
$$

for a fixed $k$ and $N \rightarrow \infty$.

\section{NUMERICAL RESULTS}

We consider a multiple-input single-output (MISO) channel with $N$ transmit antennas in Weibull fading. The PDF of the Weibull distribution can be characterized by shape parameter $\eta$ and scale parameter $\alpha$ [2]. According to [1], the normalizing constants for Weibull distribution are given by $\tilde{a}_{N}=\alpha(\ln (N))^{\frac{1}{\eta}}$ and $\tilde{b}_{N}=\frac{\alpha}{\eta}(\ln (N))^{-\frac{\eta-1}{\eta}}$, as $N \rightarrow \infty$. In Fig. 1, we plot the average throughput as a function of the number of transmit antennas, $N$, for $\eta=2$ and $\alpha=3$ and different values of $k$. We validate the obtained analytical results using Monte Carlo simulations. We observe that the asymptotic expression is accurate even for not so large $N$ while for small values of $N$ the asymptotic expression is less accurate compared to the simulations.

It is intractable to provide a mathematical characterization of the difference between the exact and the asymptotic results for the average throughput. However, numerical results show that this difference is not large even for small values of $N$ as shown in Fig. 1. For example, if we consider the worst case in which there exist three transmit antennas and we select the third best channel (the worst channel) the difference in average throughput between the asymptotic and simulation results for the case of $N=3$ and $k=3$ is approximately $0.4 \mathrm{bit} / \mathrm{s}$, while the difference for the case of $N=100$ and $k=3$ is approximately $0.025 \mathrm{bit} / \mathrm{s}$, which emphasizes that the difference vanishes asymptotically as $N$ grows large with respect to $k$.

We also notice from Fig. 1 that the gap between the asymptotic and simulation results increases as $k$ increases. This is because the asymptotic analysis is more accurate for 
large $N$ relative to a fixed $k$. Consequently, if the value of $k$ is close enough to $N$, it is expected that the asymptotic expression will be less accurate. For example, the gap between the asymptotic and simulation results for $N=5$, and $k=3$ is larger compared to the case when $N=5, k=2$ and $N=5, k=1$.

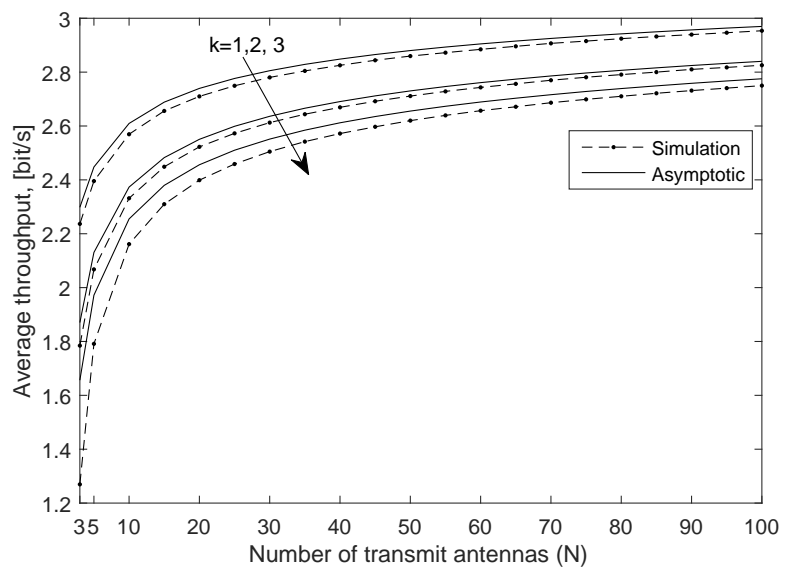

Fig. 1. Average throughput versus the number of transmit antennas $N$, for $k=1,2,3$, at $\rho=0 \mathrm{~dB}$.

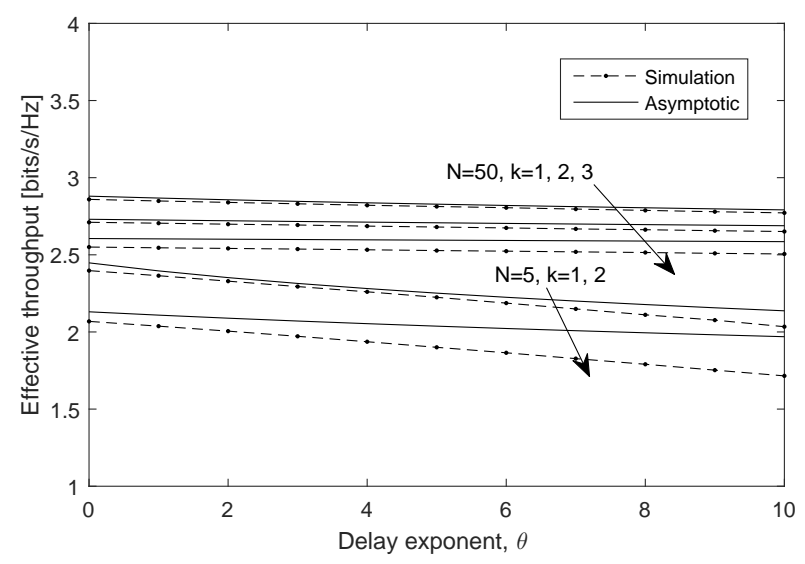

Fig. 2. Effective throughput versus delay exponent, $\theta$, for $N=5,50$ for different values of $k$ at $\rho=0 \mathrm{~dB}$.

In Fig. 2, we plot the effective throughput as a function of delay exponent, $\theta$, for $N=5$ and $N=50$, for $\eta=2$ and $\alpha=3$ and different values of $k$. We observe that for $N=5$ the derived asymptotic result is less accurate as $\theta$ increases. However, for $N=50$ the derived asymptotic results becomes very accurate and the effective throughput does not dramatically change as $\theta$ increases and it remains close to the average throughput $(\theta=0)$. This is because employing SD schemes takes advantage of the tail behavior of the fading distribution as the number of antennas increases. This emphasizes that employing SD schemes in the presence of large number of transmit antennas will combat stringent delay QoS requirements. We also observe that the asymptotic expression is less accurate as $k$ gets closer to $N$ as previously observed for the average throughput.

In Fig. 3, we plot the asymptotic average BEP as a function of the number of transmit antennas, $N$, for $\eta=2$ and $\alpha=5$ and different values of $k$. We validate the obtained analytical results using Monte Carlo simulations. We observe that the asymptotic expression is accurate even for not so large $N$.

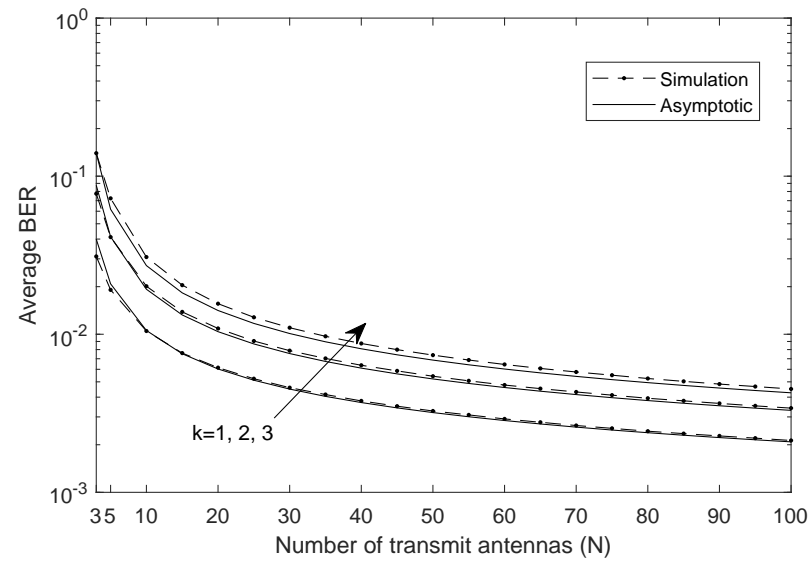

Fig. 3. Asymptotic BER of BFSK $(C=g=0.5)$ versus the number of transmit antennas $N$, for $k=1,2,3$, at $\rho=0 \mathrm{~dB}$.

\section{CONCLUSION}

We used extreme value theory to derive the asymptotic distribution of the throughput of the $k$-th best link over Weibull, Gamma, $\alpha-\mu$ and Gamma-Gamma fading channels. Using this result, we derived simple closed-form asymptotic expressions for the average throughput and effective throughput. Furthermore, we analyzed the average BEP and derived closed-form asymptotic expression for it. As a special case, we considered the Weibull fading channel model and used Monte Carlo simulations to confirm the accuracy of the derived asymptotic expressions.

\section{APPENDIX Proof Of LEMMA}

To prove the Lemma in Section III. A , we first need the following Lemma from [13].

Lemma: For any sequence $Z_{N}$ converging in distribution to a random variable $X$ that has a nondegenerate distribution function, if $E\left[\left[\left(Z_{N}\right)^{-}\right]^{p}\right]<\infty$ for any positive real number $p$, where $(x)^{-}=\max (-x, 0)$, then

$$
\lim _{N \rightarrow \infty} E\left[Z_{N}^{p}\right]=E\left[X^{p}\right],
$$

provided $E\left[|X|^{p}\right]<\infty$.

Since $R_{(N-k+1)}$ represents the $k$-th maximum of nonnegative random variables, then $E\left\{\left[\left(R_{(N-k+1)}\right)^{-}\right]^{p}\right\}<\infty$ is satisfied. However, for $E\left[\left(\frac{R_{(N-k+1)}-a_{N}}{b_{N}}\right)^{p}\right] \rightarrow E\left[X^{p}\right]$ to hold, $E\left[|X|^{p}\right]<\infty$ should be also satisfied. Let $Y=|X|$, 
where the CDF of $X$ is as expressed in (13). Then the CDF of $Y, G(y)$, can be expressed as

$$
G(y)=\sum_{j=0}^{k-1} \frac{e^{-e^{-y}} e^{-j y}-e^{-e^{y}} e^{j y}}{j !}, \quad y \geq 0,
$$

and $E\left[Y^{p}\right]$ can be expressed as

$$
\begin{aligned}
E\left[Y^{p}\right] & =p \int_{0}^{\infty} y^{p-1}[1-G(y)] d y \\
& =p \int_{0}^{\infty} y^{p-1}\left[1+\sum_{j=0}^{k-1} \frac{e^{-e^{y}} e^{j y}-e^{-e^{-y}} e^{-j y}}{j !}\right] d y .
\end{aligned}
$$

As $y \rightarrow 0$, the integrand behaves like $y^{p-1}$, using $p$-test; the integral is convergent for all $p>0$. As $y \rightarrow \infty$, the integrand behaves like $O\left(-y^{p-1} \sum_{j=1}^{k-1} e^{-j y}\right)$ and therefore the integral is convergent for all $p>0$. Hence, we conclude that $E\left[|X|^{p}\right]<\infty$ for $p>0$. To prove the rest of Lemma 2 , we need the PDF of $X, f^{(k)}(x)$. Differentiating (13), after some basic algebraic manipulation, $f^{(k)}(x)$ can be simplified as

$$
f^{(k)}(x)=\frac{e^{-k x} e^{-e^{-x}}}{(k-1) !},-\infty<x<\infty .
$$

Then, $E\left[X^{p}\right]$ can be expressed as

$$
E\left[X^{p}\right]=\int_{-\infty}^{\infty} \frac{x^{p} e^{-k x} e^{-e^{-x}}}{(k-1) !} d x .
$$

Using $u=e^{-x}$, we have

$$
E\left[X^{p}\right]=\int_{0}^{\infty} \frac{(-\ln (u))^{p} e^{-u} u^{k-1}}{(k-1) !} d u .
$$

From the definition of gamma function, we have $\Gamma(k)=$ $\int_{0}^{\infty} u^{k-1} e^{-u} d u$. By differentiating both sides with respect to $k$, where $k$ is an integer, we have $\Gamma(k) \psi(k)=$ $\int_{0}^{\infty} u^{k-1} e^{-u} \ln (u) d u$. Using this and setting $p=1$ in (33), it follows that $E[X]=-\psi(k)$. Using (31) and the transformation of $u=e^{-x}, \mathcal{M}_{X}(t)$ can be expressed as

$$
\begin{aligned}
\mathcal{M}_{X}(t) & =E\left[e^{t X}\right]=\int_{0}^{\infty} \frac{e^{-u} u^{k-t-1}}{(k-1) !} d u . \\
& =\frac{\Gamma(k-t)}{(k-1) !}, k>t .
\end{aligned}
$$

\section{ACKNOWLEDGEMENT}

This publication was made possible by the NPRP award [NPRP 8-648-2-273] from the Qatar National Research Fund (a member of The Qatar Foundation). The statements made herein are solely the responsibility of the authors.

\section{REFERENCES}

[1] H. David and H. Nagaraja, "Order Statistics," Wiley Interscience, 2003.

[2] M. K. Simon and M.-S. Alouini, Digital Communication over Fading Channels, 2nd ed. John Wiley \& Sons, Inc., 2005.

[3] S. S. Ikki and M. H. Ahmed, "On the performance of cooperativediversity networks with the Nth best-relay selection scheme," IEEE Transactions on Communications, vol. 58, no. 11, pp. 3062-3069, November 2010.

[4] X. Zhang, Z. Yan, Y. Gao, and W. Wang, "On the study of outage performance for cognitive relay networks (CRN) with the Nth best-relay selection in Rayleigh-fading channels," IEEE Wireless Communications Letters, vol. 2, no. 1, pp. 110-113, February 2013.

[5] Y. Wang and J. P. Coon, "Difference antenna selection and power allocation for wireless cognitive systems," IEEE Transactions on Communications, vol. 59, no. 12, pp. 3494-3503, December 2011.

[6] Y. Ma, J. Jin, and D. Zhang, "Throughput and channel access statistics of generalized selection multiuser scheduling," IEEE Transactions on Wireless Communications, vol. 7, no. 8, pp. 2975-2987, August 2008.

[7] H.-C. Yang, "New results on ordered statistics and analysis of minimumselection generalized selection combining (GSC)," IEEE Transactions on Wireless Communications, vol. 5, no. 7, pp. 1876-1885, July 2006.

[8] M. F. Hanif, M. Shafi, P. J. Smith, and P. Dmochowski, "Interference and deployment issues for cognitive radio systems in shadowing environments," in 2009 IEEE International Conference on Communications, June 2009, pp. 1-6.

[9] D. Wu and R. Negi, "Effective capacity: a wireless link model for support of quality of service," IEEE Transactions on Wireless Communications, vol. 2, no. 4, pp. 630-643, July 2003.

[10] Y. H. Al-Badarneh, C. N. Georghiades, and C. E. Mejia, "On the effective rate of MISO/TAS systems in Rayleigh fading," in 2017 IEEE International Symposium on Information Theory.

[11] M. S. Alouini and A. J. Goldsmith, "Capacity of Rayleigh fading channels under different adaptive transmission and diversity-combining techniques," IEEE Transactions on Vehicular Technology, vol. 48, no. 4, pp. 1165-1181, Jul 1999.

[12] M. Hanif, H. C. Yang, and M. S. Alouini, "Capacity bounds for kth best path selection over generalized fading channels," IEEE Communications Letters, vol. 18, no. 2, pp. 261-264, February 2014.

[13] G. Song and Y. Li, "Asymptotic throughput analysis for channel-aware scheduling," IEEE Transactions on Communications, vol. 54, no. 10, pp. 1827-1834, Oct 2006.

[14] D. Bai, P. Mitran, S. S. Ghassemzadeh, R. R. Miller, and V. Tarokh, "Rate of channel hardening of antenna selection diversity schemes and its implication on scheduling," IEEE Transactions on Information Theory, vol. 55, no. 10, pp. 4353-4365, Oct 2009.

[15] C. Tepedelenlioglu, Y. Zhang, and O. Rahman, "Asymptotic BER analysis of a SIMO multiuser diversity system," IEEE Transactions on Vehicular Technology, vol. 58, no. 9, pp. 5330-5335, Nov 2009.

[16] M. Hanif, H. C. Yang, and M. S. Alouini, "Receive antenna selection for underlay cognitive radio with instantaneous interference constraint," IEEE Signal Processing Letters, vol. 22, no. 6, pp. 738-742, June 2015.

[17] Y. H. Al-Badarneh, C. N. Georghiades, and C. E. Mejia, "Asymptotic performance analysis of multiuser diversity in free space optical communication systems," in 2017 IEEE Wireless Communications and Networking Conference (WCNC), March 2017, pp. 1-6.

[18] S. Al-Ahmadi, "Asymptotic capacity of opportunistic scheduling over gamma-gamma (generalized-k) composite fading channels," IET Communications, vol. 6, no. 18, pp. 3231-3237, Dec 2012.

[19] P. Chareka, "The converse to curtiss' theorem for one-sided moment generating functions," arXiv preprint arXiv:0807.3392, 2008.

[20] R.-D. Reiss, Approximate Distributions of Order Statistics: With Applications to Nonparametric Statistics. Springer Science \& Business Media, 2012 